\title{
Supramolecular assembly of KAT2A with succinyl-CoA for histone succinylation
}

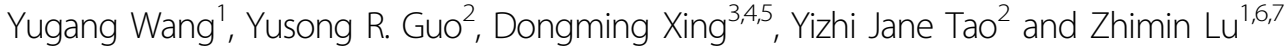

\section{Dear Editor,}

Histone modifications regulate many fundamental biological processes, including DNA replication, transcription, and repair. Eighteen posttranslational modifications of histones, including acetylation, succinylation, and methylation, have been reported ${ }^{1-3}$. Lysine acetyltransferase 2A (KAT2A, also known as GCN5), a member of the GCN5-related N-acetyltransferase superfamily and a component of Spt-Ada-KAT2A-acetyltransferase (SAGA) and Ada-two-A-containing complexes, was identified as the first transcription-related histone acetyltransferase in $1996^{4,5}$. KAT2A binds to acetylcoenzyme A (CoA) and transfers its acetyl group to histones to regulate chromatin architecture and locusspecific transcription ${ }^{6}$.

Our recent studies reported that KAT2A can also function as a histone succinyltransferase by directly transferring the succinyl group from succinyl-CoA to histone H3 lysine 79 (H3K79), which is important for the regulation of gene expression in tumor cells ${ }^{7}$. To elucidate the catalytic mechanism, we determined the structures of both the apo and succinyl-CoA-complexed KAT2A using $\mathrm{X}$-ray crystallography ${ }^{7}$. By comparing these structures with that of KAT2A in complex with acetyl-CoA ${ }^{7}$, we previously demonstrated that succinyl-CoA and acetylCoA occupy similar binding sites in the catalytic domain of KAT2A and identified key residues interacting with the acyl chains ${ }^{7}$. In the present work, we report a novel highorder assembly for the catalytic domain of KAT2A observed in the crystal structures of both the apo and succinyl-CoA complexes. It is important to note that both

\footnotetext{
Correspondence: Yizhi Jane Tao (ytao@rice.edu) or

Zhimin Lu (zhiminlu@mdanderson.org)

${ }^{1}$ Brain Tumor Center, Department of Neuro-Oncology, The University of Texas MD Anderson Cancer Center, Houston, TX 77030, USA

${ }^{2}$ Department of BioSciences, Rice University, Houston, TX 77005, USA

Full list of author information is available at the end of the article.

These authors contributed equally: Yugang Wang, Yusong R. Guo
}

crystals were grown under conditions ( $\mathrm{pH}$ of 4.6) different from the previously reported crystallization condition $(\mathrm{pH}$ of 7.0) of a monomer of the catalytic domain of KAT2 ${ }^{8}$.

Although apo and succinyl-CoA-complexed KAT2A were crystallized in different space groups with different crystal packing contacts (i.e., $\mathrm{P} 4_{1} 2_{1} 2$ vs. $\mathrm{P} 2{ }_{1} 3$ ), the same octahedral supramolecular assembly was observed in both crystals: 24 KAT2A molecules formed a spherically shaped complex with a hollow center (Fig. 1a-c). Octahedral assemblies are characterized by 4 -fold, 3 -fold, and 2 -fold symmetry axes. With these symmetry operations, an assembly of 24 molecules is generated from a single protein protomer. In the crystal of apo-KAT2A (i.e., space group $\left.\mathrm{P}_{1} 2_{1} 2\right), 24$ molecules in each crystallographic asymmetric unit gave rise to a complete octahedral assembly. The crystal of succinyl-CoA-complexed KAT2A had the space group of $\mathrm{P} 2{ }_{1} 3$ with eight molecules in each asymmetric unit, but 24 molecules from three asymmetric units made a complete octahedron of the KAT2A-succinyl CoA complex (Fig. 1a-c).

The stability of a protein-protein interaction usually correlates with the amount of buried surface areas. Based on results from an interface analysis performed by the program PISA ${ }^{9}$, we found that the formation of the KAT2A octahedron structure was mediated by strong interactions at both dimer and tetramer interfaces, with total buried surface areas of $1400 \AA^{2}$ and $1300 \AA^{2}$, respectively (Fig. 1d-f; Supplementary Table S1). At the dimer interface, helices $\alpha 1$ and $\alpha 2$ from one subunit were in close contact with the same two helices from a neighboring subunit (Fig. 1d). The formation of tetramers in the octahedron was largely mediated by $\alpha 2, \alpha 7$, and loop 3 of one molecule interacting with $\alpha 4$ and the $C$ terminal tail of an adjacent molecule (Fig. 1e). Interactions of KAT2A around the three-fold symmetry axis were weak; the buried surface area was less than $10 \AA^{2}$ (Fig. 1f), suggesting that tetramers or dimers may act as precursors 


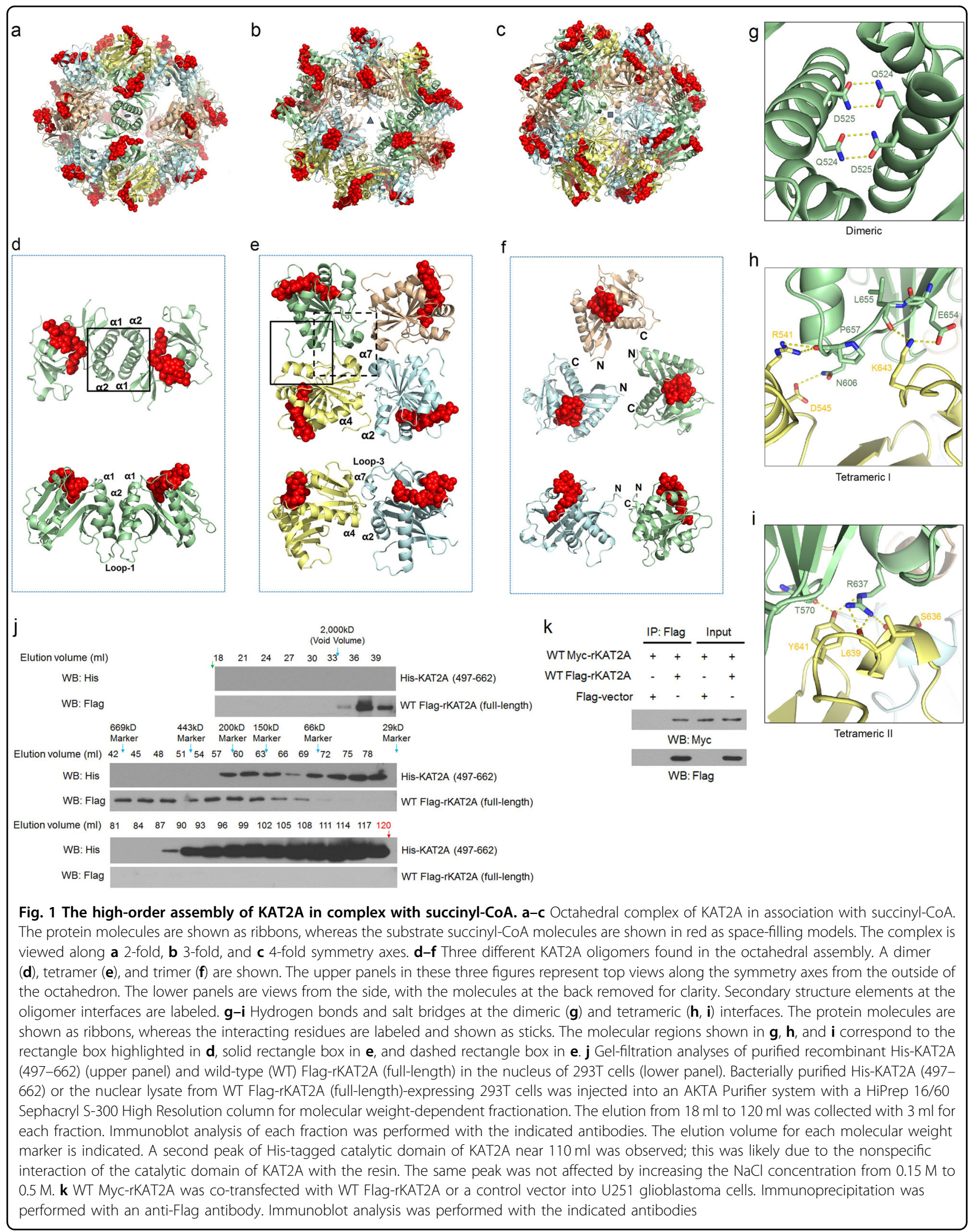


for octahedron assembly. Interactions at the dimer interface were mostly hydrophobic in nature, except for two pairs of hydrogen bonds mediated by Q524 and D525 (Fig. 1g). In comparison, more polar interactions were observed at the tetrameric interface, including a salt bridge mediated by E654 and K643, as well as hydrogen bonds mediated by the side chains of R541, D545, R637, and Y641 (Fig. 1h, i).

To determine the physiological relevance of the octahedral structure of KAT2A, we employed gel-filtration analyses and identified high-molecular weight complexes of the purified catalytic domain of KAT2A, ranging from $\sim 29 \mathrm{kD}$ to the fractions that correspond to the molecular weight larger than $200 \mathrm{kD}$ (Fig. 1j). Full-length KAT2A isolated from nuclear fractions of 293T cells gave rise to even larger complexes, with an estimated molecular weight of $94 \mathrm{kD}$, up to a maximal detectable molecular weight $(\geq 2000 \mathrm{kD}$; Fig. $1 \mathrm{j})$. The wide range of molecular weight distributions may include assembly intermediates expected for an octahedral supramolecular complex. Additionally, immunoprecipitation analysis of cells expressing both full-length Flag-tagged KAT2A and full-length Myc-tagged KAT2A confirmed interaction between individual KAT2A proteins (Fig. 1k), further supporting the notion that KAT2A forms high-order assemblies in cells.

Close inspection of the crystal structure of the catalytic domain of KAT2A indicated that both $\mathrm{N}$ - and C-termini are exposed on the outer surface of the octahedron (Supplementary Fig. S1). Therefore, other components of the catalytic complex (i.e., the PCAF-HD and bromodomain of KAT2A) may connect to the catalytic domain of KAT2A and may be situated on the surface of the octahedron without disturbing its overall structural organization. Furthermore, all 24 substrate-binding pockets in the octahedron structure are exposed externally (Fig. 1d-f). Thus, rapid diffusion of either acetyl-CoA or succinyl-CoA in and out of the KAT2A active site is possible during catalysis.

Although yet to be reported for any histone acetyltransferases, supramolecular assemblies of enzymes frequently form and are considered advantageous in capturing substrates ${ }^{10}$. Similarly, the formation of a spherically shaped supramolecular complex with 24 catalytic domains of KAT2A is likely to play such a role. The $\alpha$-ketoglutarate dehydrogenase $(\alpha-\mathrm{KGDH})$ complex binds to KAT2A in the nucleus and facilitates KAT2A's succinyltransferase activity by providing local succinylCoA, which can overcome the disadvantage of limited succinyl-CoA concentration in the nucleus ${ }^{7}$. With an octahedral assembly of the KAT2A: $\alpha-\mathrm{KGDH}$ complex, the substrate-binding site of KAT2A is greatly enriched, thus further facilitating the capture of $\alpha-K G D H-$ produced succinyl-CoA by KAT2A to ensure efficient
H3K79 succinylation in vivo. Thus, the supramolecular assemblies of the catalytic domain of KAT2A, high local concentrations of succinyl-CoA generated by the KAT2A-associated $\alpha-\mathrm{KGDH}$ complex, and high catalytic activity of KAT2A toward succinyl-CoA ${ }^{7}$ should help compensate for the relatively low nuclear concentration of succinyl-CoA to promote KAT2A-mediated histone succinylation.

Together, our recent publication ${ }^{7}$ and these new structural results for the first time identified a histone succinyltransferase, KAT2A, which was known as a histone acetyltransferase. In addition, we unearthed an important mechanism of histone $\mathrm{H} 3$ succinylation, in which KAT2A uses succinyl-CoA locally generated by $\alpha$ $\mathrm{KGDH}$ in the same supramolecular complex to posttranslationally modify histone $\mathrm{H} 3$ for gene transcription. Our findings highlight the significance of the metabolic enzyme $\alpha$-KGDH-coupled KAT2A complex in the regulation of gene expression, tumor cell proliferation, and tumor formation.

\section{Acknowledgements}

We thank Li Li at The University of Texas Health Science at Houston for technical support and Erica Goodoff for critical reading of this manuscript. This work was supported by National Institute of Neurological Disorders and Stroke grant R01 NS089754 (to Z.L.), NCl grant 1R01 CA204996 (to Z.L.), the NIH/NCl P30 CA016672 (to Z.L.), Welch Foundation grant C-1565 (to Y.J.Y.), and the 2018 UT Proteomics Network Pilot Fund (to Y.W.). Z.L. is a Ruby E. Rutherford Distinguished Professor.

\section{Author details}

${ }^{1}$ Brain Tumor Center, Department of Neuro-Oncology, The University of Texas MD Anderson Cancer Center, Houston, TX 77030, USA. ${ }^{2}$ Department of BioSciences, Rice University, Houston, TX 77005, USA. ${ }^{3}$ Cancer Institute, The Affiliated Hospital of Qingdao University, Qingdao, Shandong, China. ${ }^{4}$ Qingdao Cancer Institute, Qingdao 266061 Shandong, China. ${ }^{5}$ School of Life Sciences, Tsinghua University, 100084 Beijing, China. ${ }^{6}$ Department of Molecular and Cellular Oncology, The University of Texas MD Anderson Cancer Center, Houston, TX 77030, USA. ${ }^{7}$ Graduate School of Biomedical Sciences, Houston, TX 77030, USA

\section{Authors' contributions}

Z.L., Y.T., D.X., Y.R.G., and Y.W. conceived and designed the study and wrote the manuscript. Y.W. and Y.R.G. performed the experiments.

\section{Conflict of interest}

The authors declare that they have no conflict of interest.

Supplementary Information accompanies the paper at (https://doi.org/ 10.1038/s41421-018-0048-8).

Received: 1 March 2018 Revised: 10 May 2018 Accepted: 15 June 2018 Published online: 07 August 2018

\footnotetext{
References

1. Tan, M. et al. Identification of 67 histone marks and histone lysine crotonylation as a new type of histone modification. Cell 146, 1016-1028 (2011).

2. Huang, H., Sabari, B. R., Garcia, B. A., Allis, C. D. \& Zhao, Y. SnapShot: histone modifications. Cell 159, 458-458.e1 (2014).
} 
3. Tessarz, P. \& Kouzarides, T. Histone core modifications regulating nucleosome structure and dynamics. Nat. Rev. Mol. Cell Biol. 15, 703-708 (2014).

4. Brownell, J. E. et al. Tetrahymena histone acetyltransferase A: a homolog to yeast Gcn5p linking histone acetylation to gene activation. Cell $\mathbf{8 4}, \mathbf{8 4 3 - 8 5 1}$ (1996).

5. Grant, P. A. et al. Yeast Gen5 functions in two multisubunit complexes to acetylate nucleosomal histones: characterization of an Ada complex and the SAGA (Spt/Ada) complex. Genes Dev. 11, 1640-1650 (1997).

6. Lin, Y., Fletcher, C. M., Zhou, J., Allis, C. D. \& Wagner, G. Solution structure of the catalytic domain of GCN5 histone acetyltransferase bound to coenzyme A. Nature 400, 86-89 (1999).
7. Wang, Y. et al. KAT2A coupled with the alpha-KGDH complex acts as a histone H3 succinyltransferase. Nature 552, 273-277 (2017).

8. Schuetz, A. et al. Crystal structure of a binary complex between human GCN5 histone acetyltransferase domain and acetyl coenzyme A. Proteins 68, 403-407 (2007).

9. Krissinel, E. \& Henrick, K. Inference of macromolecular assemblies from crystalline state. J. Mol. Biol. 372, 774-797 (2007).

10. Persson, K., Schneider, G., Jordan, D. B., Viitanen, P. V. \& Sandalova, T. Crystal structure analysis of a pentameric fungal and an icosahedral plant lumazine synthase reveals the structural basis for differences in assembly. Protein Sci. $\mathbf{8}$, 2355-2365 (1999). 